\title{
PERSPECTIVE OPEN A technology-assisted life of recovery from psychosis
}

\author{
Dror Ben-Zeev ${ }^{1}$, Benjamin Buck ${ }^{1}$, Sarah Kopelovich ${ }^{1}$ and Suzanne Meller ${ }^{1}$
}

Developments in digital health technologies have the potential to expedite and strengthen the path towards recovery for people with psychosis. This perspective piece provides a snapshot of how a range of digital technologies can be deployed to support a young adult's efforts to cope with schizophrenia-spectrum illness. In conjunction with a day in the life of this individual, we provide examples of innovations in digital health research designed for this clinical population, as well as brief summaries of the evidence supporting the usability, feasibility, or effectiveness of each approach. From early detection to ongoing symptom management and vocational rehabilitation, this day-in-the-life vignette provides an overview of the ways in which digital health innovations could be used in concert to augment, scaffold, and enhance schizophrenia-spectrum illness management and recovery.

npj Schizophrenia (2019)5:15

; https://doi.org/10.1038/s41537-019-0083-y

\section{INTRODUCTION}

Imagine a future in which the exciting digital health and computational psychiatry breakthroughs we are seeing today lead to the development of effective technology-assisted illness detection, monitoring, and treatment-support tools of tomorrow. ${ }^{1-3}$ Public-private partnerships, rapid commercialization models, and new regulatory frameworks will create opportunities to make these instruments widely available and affordable. "Digital natives" ${ }^{5}$-individuals who grew up in the internet and smartphone era-will be fully capable of using familiar technologies for personal health support. ${ }^{6,7}$ Our scientific understanding of psychopathology will progress to a point that we may no longer view psychosis as a fixed and hopeless state but as a continuum of experience that varies both across people and within the same individual over time. ${ }^{8,9}$ When these conditions converge, a range of technology-based and technology-assisted mental health resources will provide individuals with psychosis more opportunities to actualize their potential for recovery. In what follows, we describe what the daily life of a young adult in recovery from psychosis may look like in what we believe to be the not-toodistant future.

Annie is a 21-year-old woman in her junior year of college. She works part-time at a flower shop in her neighborhood, owns a 4month-old energetic puppy that she adopted from the local animal shelter, and is in a committed relationship with her boyfriend of two years. Annie began to experience depressed mood and derogatory auditory hallucinations just over a year ago. Feeling anxious about her wellbeing, she Googled "where do voices come from," "hearing whispers," and "do I have schizophrenia". [The Pew Research Center found that one in three American adults have gone online to try to understand a health condition. ${ }^{10}$ Google AdWords have been employed as part of a digital outreach campaign, resulting in over four thousand clickthroughs to psychosis-specific psychoeducation, psychosis selfscreening, and encouragement to reach out to local early psychosis clinical services. ${ }^{11}$ ] She completed an online self-report mental health screener that alerted her to a need for additional assessment. [An online-administered screener for psychosis has demonstrated positive predictive value that exceeded that of clinician-administered interview in estimating psychosis conversion. ${ }^{12}$ Risk calculators incorporating demographics, family history and other clinical and functional indicators have demonstrated promise and are similarly available online. ${ }^{13}$ ] As a part of this follow-up assessment, she recorded several prompted speech samples using her laptop, [Natural Language Processing (NLP) systems with automated machine learning ${ }^{14,15}$ have demonstrated promise in strengthening predictions of psychosis risk. ${ }^{16}$ One approach deployed in a high-risk sample demonstrated cross-validated accuracy predicting psychosis onset. $\left.{ }^{17}\right]$ and received neuroimaging at a nearby testing center. [Machine-learning analytics of functional imaging data generated correct identification of schizophrenia in a majority of newly diagnosed individuals. It could further predict which patients would respond to treatment with antipsychotic medication. ${ }^{18}$ ]

The early psychosis specialty clinic to which Annie was referred was nearly two hours' drive by car. Her parents took the day off of work to drive Annie to her appointment. The intake specialist reviewed her online assessments and NLP results prior to her intake appointment, which permitted him to focus more on discussing her and her parents' concerns, strengths, resources, and aspirations through the use of a web-based clinician support tool aimed to enhance shared decision-making practices. [Computerized clinician support tools may enhance delivery of best- and evidence-based practices. ${ }^{19}$ ] Annie was also able to receive a tour of the clinic, where she learned that she would be able to access her care team virtually, was introduced to her personalized digital health dashboard, received a brief orientation to her personalized menu of digital health tools, and received her first telehealth appointment for the following day. Annie and her parents were relieved that she would not need to travel back to the clinic for this appointment. ${ }^{20,21}$

Like most of the adult population, including people with severe psychiatric conditions, Annie owns a mobile phone. ${ }^{22,23}$ In the 12 months since she established care, this personal device has become her primary mental health support system. Every morning Annie receives a tailored text message that reminds her to take

\footnotetext{
${ }^{1}$ Department of Psychiatry and Behavioral Sciences, University of Washington School of Medicine, Seattle, WA, USA
}

Correspondence: Dror Ben-Zeev (dbenzeev@uw.edu)

Received: 12 August 2019 Accepted: 29 August 2019

Published online: 18 September 2019 
her antipsychotic medications. [Multiple approaches using text messages to remind users about their medications have been deployed and appear feasible and highly usable among people with serious mental illness. ${ }^{24,25}$ ] While she is eating breakfast Annie receives an instant message from her community-based case manager who reminds her that her driving exam is next week and that her rent is due. [A study of "hovering" treatment via text messages demonstrated that participants with serious mental illness respond to the majority of clinical messages sent by their community-based mobile interventionist and most find this intervention useful. ${ }^{26}$ ] Computer-based cognitive remediation training has improved her ability to focus and she was able to study the Department of Motor Vehicle's driver manual without difficulty. [Meta-analyses suggest that cognitive remediation interventions demonstrate positive medium effects for cognitive performance and functioning and small effects for symptoms. ${ }^{27,28}$ ]

Annie has a work shift before her afternoon classes. She found her job at the flower shop with the aid of a community-based supported employment specialist who also introduced her to a software program that helped her develop the skills and confidence necessary to pass the job interview successfully. [Virtual reality job interview training has been shown to improve job interview role-play performance and real-world job attainment in a randomized controlled trial. $\left.{ }^{29}\right]$ The same specialist provides her with on-the-job follow-along support regularly via a supported employment app she uses to cope with job-related challenges and stress. [A feasibility study of a mobile application designed to support the employment of individuals with serious mental illness in real time found that the majority of users found the app to be userfriendly and helpful in providing on-the-job supports. ${ }^{30}$ ] She completes her shift and heads over to campus for class. Before entering the lecture hall, she experiences heightened anxiety coupled with auditory hallucinations that berate her, saying that she is not smart enough to understand this subject. This is not an uncommon experience for Annie. Fortunately, she has adopted effective technology-supported coping strategies; she finds a private spot, takes out her smartphone, and activates her illness self-management app. Annie views several videos that guide her through a sequence of relaxation strategies and cognitive restructuring techniques focused on dysfunctional beliefs linked with the experience of hearing voices. [A smartphone selfmanagement intervention called FOCUS has been shown to be usable, ${ }^{31}$ feasible, ${ }^{32}$ engaging, and clinically effective. ${ }^{33}$ ] In just a few minutes she feels calmer and is able to find her seat and fully engage in the class. She activates her smartphone to audio record today's lecture.

Picking up her phone after class, Annie notices that she has an encouraging text message from her mother. Over the last few months, her mom has been participating in an online group of family members of young adults with psychosis. Since she joined, Annie has noticed that her parents are less anxious when Annie talks about her symptoms. As a result, Annie has been more open and their relationship has improved. [Proof-of-concept studies have demonstrated that online psychoeducation for individuals with schizophrenia and their family members is feasible and acceptable. ${ }^{34}$ A web-based family and client psychoeducation intervention has demonstrated significant improvements in positive symptoms and knowledge about schizophrenia. ${ }^{35}$ ] Leaving class, she checks the likelihood of traffic on her commute on her phone. Because walking through crowds increases her anxiety and paranoia, she had been previously intentionally avoiding crowds on her commute. However, today, she decides to take the quickest (but also busiest) way home. Over the past few weeks, she has been challenging her beliefs about the dangerousness of crowds with a virtual reality (VR) self-training tool, and she decides today's the day she's going to give this route a shot. [Virtual reality cognitive therapy for persecutory ideation has been shown to reduce delusional belief conviction and associated distress. ${ }^{36,37}$ ]
When Annie returns home from class, she grabs a handful of pretzels and a soda from the fridge. She has an online session scheduled with her therapist just before dinner and wants to snack before the call. [Individuals with psychosis use and are satisfied with clinical services delivered via two-way videoconferencing. ${ }^{38}$ ] As she drinks her soda, she fills out a brief outcome questionnaire on her phone that will be reviewed by her clinician ahead of their session. In the free-text window, she writes that she walked through the busiest parts of campus today and it went very well. [Mobile apps deploying remote outcome assessments appear feasible and acceptable in specialty early psychosis settings, and generate ratings that are similar to gold-standard clinical tools. $^{39}$ Such systems provide opportunities for monitoring and personally tailored care.] She is excited to share the news about her successful exposure with her clinician later that evening. During the session, her therapist displays to Annie her symptom assessment scores over the last four months of treatment. Annie notices that her anxiety has diminished since engaging in exposure treatment, and her voices have also become less intense and malevolent. ${ }^{40}$ Annie and her therapist reflect on this pattern and discuss the coping strategies that seemed to have helped the most. Guided by her therapist, Annie selects coping strategies that she will employ to help self-regulate distress during her upcoming driver's test. Next, Annie and her therapist continue to treat her voices-related distress. Annie's therapist presents her voice avatar on the screen, so that Annie can become more comfortable tolerating the voices and practice rational responses when the voice content is derogatory. [A computerized treatment has shown therapeutic effects on auditory verbal hallucination severity by encouraging individuals to engage in a dialogue with digital representations of their voices. ${ }^{41}$ This work has been extended in a randomized trial. ${ }^{42}$ More immersive, virtual reality platforms have also demonstrated positive effects. ${ }^{43}$ ]

Because Annie's therapist is still in a Cognitive Behavioral Therapy for Psychosis (CBTp) training program, Annie has consented to have their sessions recorded and reviewed by a CBTp-trained supervisor. When the pair log off, the session is uploaded to a remote server so that her therapist can receive feedback from her supervisor. ${ }^{44}$ In addition to personalized, specific, and prompt feedback on her competence and adherence, Annie's therapist can virtually access continual education, support, and telementoring via an online portal. [Web-based training, followed by peer-led consultation with previously-trained providers appears to be a viable way to enhance knowledge acquisition and competency. ${ }^{45}$ Telementoring among community mental health clinicians learning CBTp enhances confidence and affects their approach with patients. ${ }^{46}$ ]

Meanwhile, after logging off from her therapy session, Annie updates her online early psychosis community with a post to the group feed. She had been sharing her experiences with peers online and several others had encouraged her to try the VR program. She is excited to let them know that it was helpful! [An online social community for young adults with early psychosis has been examined in a feasibility study, revealing high system usage over a 3-week period. ${ }^{47}$ ] Annie closes her laptop and takes a look at her phone. She sees a calendar reminder that she's scheduled to meet with friends for dinner and a movie.

On the way to the restaurant, she passes several liquor stores. In her pocket, her phone generates several new notifications reminding her that alcohol can exacerbate her symptoms. She remembers that a few years ago, when she was starting college and struggling with significant depression, she was drinking heavily. [A mobile app supports the recovery of individuals with substance abuse concerns by providing them with information and support as well as a monitoring feature that prompts app users when they are near high-risk locations as detected by GPS. ${ }^{48}$ ] Annie has a great time at dinner and at the movie with her friends, and after the movie ends, like so many other Americans, she uses her 
rideshare app to request a ride home. While she is waiting for her car to arrive she kills time by watching today's vlog update by an individual with lived experience she has been following who posts his recovery testimonials on YouTube. [Survey studies suggest that young adults with early psychosis often turn to YouTube as a primary source for information and virtual community support related to mental illness. ${ }^{49,50}$ ] She chuckles as she reads some of the comments and is reminded how so many like her with a serious mental illness not only cope, but also thrive.

\section{DISCUSSION}

The peer-reviewed literature is now replete with descriptions of novel digital health approaches for help-seeking individuals with psychosis. The evidence suggests that individuals with psychosis use electronic devices, digital applications, and social media resources in a manner that is comparable to those without psychosis. Annie's story paints a picture of how a young digital native may use a variety of technologies to improve their quality of life, extend symptom management into their day-to-day activities, and facilitate wellness. Many of the tools we reference in the vignette are in early phases of development (e.g., proof of concept, feasibility), and more rigorous testing is needed to ascertain for whom and in what contexts they are most suitable. Future developments will not only determine the specific components of these interventions, but how best to integrate them with existing mental health services.

The ubiquity and increasing utility of technology in our everyday lives may concern those who fear the overreliance on technology, privacy breaches, or that digital health technologies will replace human-delivered assessment and treatment. These concerns must be balanced with the potential for technology to enhance care and healthy practices, whether employed independently or with the assistance of a care team. The coordinated specialty care (CSC) model has transformed the standard of inperson care for first-episode psychosis, emphasizing a teambased, multi-element approach to address the complex and varied needs of this population. ${ }^{51}$ Still, significant barriers, including cost, workforce development, and community involvement remain in expanding access to these interventions. ${ }^{52}$ As demonstrated in Annie's narrative, technology can be additive to human-delivered, evidence-based treatment. These technologies are consistent with the multi-component emphasis of CSC, as they connect her with others to create a team of community case managers, employment specialists, therapists, and people with lived experience that can help her succeed.

In many respects, this "day-in-the-life" is an ideal. In a typical day there are many opportunities for an individual experiencing psychosis to either engage in protective or risky behaviors. Over time, these coping skills have cumulative effects and important implications for mental health, functioning, self-determination, and quality of life. Emerging technologies may serve as the key scaffold to implementing effective practices in a scalable, personalized, and sustainable manner. While we can imagine this future today, the developments of tomorrow will determine whether we realize this vision.

\section{DATA AVAILABILITY}

No original data were used in the preparation of this article.

\section{AUTHOR CONTRIBUTIONS}

All authors worked on the conceptualization of this perspective piece, conducted literature reviews, contributed written content, edited, and formatted the article in preparation for publication.

\section{ADDITIONAL INFORMATION}

Competing interests: Ben-Zeev owns the IP for the FOCUS intervention that is described in an article referenced in this paper. He has an intervention content licensing agreement with Pear Therapeutics and has consulted for eQuility and Otsuka. The remaining authors declare no competing interests.

Publisher's note Springer Nature remains neutral with regard to jurisdictional claims in published maps and institutional affiliations.

\section{REFERENCES}

1. O'Hanlon, P. et al. Tomorrow's world: current developments in the therapeutic use of technology for psychosis. BJPsych. Adv. 22, 301-310 (2016).

2. Hollis, $C$. et al. Technological innovations in mental healthcare: harnessing the digital revolution. Br. J. Psychiatry 206, 263-265 (2015).

3. Mohr, D. C., Burns, M. N., Schueller, S. M., Clarke, G. \& Klinkman, M. Behavioral intervention technologies: Evidence review and recommendations for future research in mental health. Gen. Hosp. Psychiatry 35, 332-338 (2013).

4. Ben-Zeev, D. Mobile health for all: public-private partnerships can create a new mental health landscape. JMIR Ment. Heal. 3, e26 (2016),

5. Marc, P. Digital natives, digital immigrants Part 1. Horiz 9, 1-6 (2001).

6. Lal, S., Dell'Elce, J. \& Malla, A. K. Technology access and use among young adults with a first episode of psychosis. Psychiatr. Serv. 66, 764-765 (2015).

7. Abdel-Baki, A., Lal, S., D.-Charron, O., Stip, E. \& Kara, N. Understanding access and use of technology among youth with first-episode psychosis to inform the development of technology-enabled therapeutic interventions. Early Interv. Psychiatry 11, 72-76 (2017).

8. Cuthbert, B. N. The RDoC framework: facilitating transition from ICD/DSM to dimensional approaches that integrate neuroscience and psychopathology. World Psychiatry 13, 28-35 (2014).

9. Insel, $T$. et al. Research Domain Criteria (RDoC): toward a new classification framework for research on mental disorders. Am. J. Psychiatry 167, 748-751 (2010).

10. Fox, S. \& Duggan, M. Health Online 2013. Pew Internet and American Life Project. https://www.pewinternet.org/2013/01/15/health-online-2013/.

11. Birnbaum, M. L. et al. Using digital media advertising in early psychosis intervention. Psychiatr. Serv. 68, 1144-1149 (2017).

12. Brodey, B. B. et al. The Early Psychosis Screener for Internet (EPSI)-SR: Predicting 12 month psychotic conversion using machine learning. Schizophr. Res. 208, 390-396 (2019).

13. Cannon, T. D. et al. An individualized risk calculator for research in prodromal psychosis. Am. J. Psychiatry 173, 980-988 (2016).

14. Bedi, G. et al. Automated analysis of free speech predicts psychosis onset in highrisk youths. Npj Schizophr. 1, 15030 (2015).

15. Rezaii, N., Walker, E. \& Wolff, P. A machine learning approach to predicting psychosis using semantic density and latent content analysis. NPJ Schizophr. 5, 9 (2019).

16. Corcoran, C. M., Benavides, C. \& Cecchi, G. A. Natural language processing: opportunities and challenges for patients, providers, and hospital systems. Psychiatr. Ann. 49, 202-208 (2019).

17. Corcoran, C. M. et al. Prediction of psychosis across protocols and risk cohorts using automated language analysis. World Psychiatry 17, 67-75 (2018).

18. Cao, B. et al. Treatment response prediction and individualized identification of first-episode drug-naïve schizophrenia using brain functional connectivity. Mol. Psychiatry (2018). https://doi.org/10.1038/s41380-018-0106-5. [Epub ahead of print].

19. van der Krieke, L., Emerencia, A. C., Aiello, M. \& Sytema, S. Usability evaluation of a web-based support system for people with a schizophrenia diagnosis. J. Med. Internet Res. 14, e24 (2012).

20. Pfeiffer, P. N. et al. Impact of distance and facility of initial diagnosis on depression treatment. Health Serv. Res. 46, 768-786 (2011).

21. Fortney, J., Rost, K., Zhang, M. \& Warren, J. The impact of geographic accessibility on the intensity and quality of depression treatment. Med. Care 37, 884-893 (1999).

22. Ben-Zeev, D. Mobile technologies in the study, assessment, and treatment of schizophrenia. Schizophr. Bull. 38, 384-385 (2012).

23. Firth, J. et al. Mobile phone ownership and endorsement of "mHealth" among people with psychosis: a meta-analysis of cross-sectional studies. Schizophr. Bull. 42, 448-455 (2015)

24. Kannisto, K. A., Adams, C. E., Koivunen, M., Katajisto, J. \& Välimäki, M. Feedback on SMS reminders to encourage adherence among patients taking antipsychotic medication: A cross-sectional survey nested within a randomised trial. BMJ Open 5, e008574 (2015).

25. Montes, J. M., Medina, E., Gomez-Beneyto, M. \& Maurino, J. A short message service (SMS)-based strategy for enhancing adherence to antipsychotic medication in schizophrenia. Psychiatry Res. 200, 89-95 (2012). 
26. Ben-Zeev, D., Kaiser, S. M. \& Krzos, I. Remote "hovering" with individuals with psychotic disorders and substance use: feasibility, engagement, and therapeutic alliance with a text-messaging mobile interventionist. J. Dual Diagn. 10, 197-203 (2014)

27. McGurk, S. R., Twamley, E. W., Sitzer, D. I., McHugo, G. J. \& Mueser, K. T. A metaanalysis of cognitive remediation in schizophrenia. Am. J. Psychiatry 164, 1791-1802 (2007)

28. Wykes, T., Huddy, V., Cellard, C., McGurk, S. R. \& Czobor, P. A meta-analysis of cognitive remediation for schizophrenia: Methodology and effect sizes. Am. J. Psychiatry 168, 472-485 (2011).

29. Smith, M. J. et al. Virtual reality job interview training and 6-month employment outcomes for individuals with schizophrenia seeking employment. Schizophr. Res. 166, 86-91 (2015).

30. Nicholson, J., Wright, S. M., Carlisle, A. M., Sweeney, M. A. \& McHugo, G. J. The WorkingWell mobile phone app for individuals with serious mental illnesses: Proofof-concept, mixed-methods feasibility study. JMIR Ment. Heal. 5, e11383 (2018).

31. Ben-Zeev, D. et al. Development and usability testing of FOCUS: A smartphone system for self-management of schizophrenia. Psychiatr. Rehabilitation J. 36, 289-296 (2013)

32. Ben-Zeev, D. et al. Feasibility, acceptability, and preliminary efficacy of a smartphone intervention for schizophrenia. Schizophr. Bull. 40, 1244-1253 (2014).

33. Ben-Zeev, D. et al. Mobile health (mHealth) versus clinic-based group intervention for people with serious mental illness: a randomized controlled trial. Psychiatr. Serv. 69, 978-985 (2018)

34. Glynn, S. M., Randolph, E. T., Garrick, T. \& Lui, A. A proof of concept trial of an online psychoeducational program for relatives of both veterans and civilians living with schizophrenia. Psychiatr. Rehabilitation J. 33, 278-287 (2010).

35. Rotondi, A. J. et al. Web-based psychoeducational intervention for persons with schizophrenia and their supporters: one-year outcomes. Psychiatr. Serv. 61, 1099-1105 (2010)

36. Freeman, D. Studying and treating schizophrenia using virtual reality: a new paradigm. Schizophr. Bull. 34, 605-610 (2008).

37. Freeman, D. et al. Virtual reality in the treatment of persecutory delusions: randomised controlled experimental study testing how to reduce delusional conviction. Br. J. Psychiatry 209, 62-67 (2016).

38. Hulsbosch, A. M., Nugter, M. A., Tamis, P. \& Kroon, H. Videoconferencing in a mental health service in The Netherlands: a randomized controlled trial on patient satisfaction and clinical outcomes for outpatients with severe mental illness. J. Telemed. Telecare 23, 513-520 (2016).

39. Niendam, T. A. et al. Enhancing early psychosis treatment using smartphone technology: a longitudinal feasibility and validity study. J. Psychiatr. Res. 96, 239-246 (2018).

40. Delgadillo, J. et al. Improving the efficiency of psychological treatment using outcome feedback technology. Behav. Res. Ther. 99, 89-97 (2017).

41. Leff, J., Williams, G., Huckvale, M. A., Arbuthnot, M. \& Leff, A. P. Computer-assisted therapy for medication-resistant auditory hallucinations: Proof-of-concept study. Br. J. Psychiatry 202, 428-433 (2013).
42. Craig, T. K. et al. AVATAR therapy for auditory verbal hallucinations in people with psychosis: a single-blind, randomised controlled trial. Lancet Psychiatry 5, 31-40 (2018).

43. du Sert, O. P. et al. Virtual reality therapy for refractory auditory verbal hallucinations in schizophrenia: a pilot clinical trial. Schizophr. Res. 197, 176-181 (2018).

44. Dorsey, S. et al. Improving practice in community-based settings: a randomized trial of supervision - study protocol. Implement. Sci. 8, 89 (2013).

45. German, R. E. et al. Testing a web-based, trained-peer model to build capacity for evidence-based practices in community mental health systems. Psychiatr. Serv. 69, 286-292 (2017).

46. Kopelovich, S. L. et al. Statewide implementation of cognitive behavioral therapy for psychosis through a learning collaborative model. Cogn. Behav. Pract. 26, 439-452 (2019).

47. Alvarez-Jimenez, M. et al. On the HORYZON: moderated online social therapy for long-term recovery in first episode psychosis. Schizophr. Res. 143, 143-149 (2013).

48. Gustafson, D. H. et al. A smartphone application to support recovery from alcoholism: a randomized clinical trial. JAMA Psychiatry 71, 566-572 (2014).

49. Lal, S. et al. Preferences of young adults with first-episode psychosis for receiving specialized mental health services using technology: a survey study. JMIR Ment. Heal. 2, e18 (2015).

50. Naslund, J. A., Grande, S. W., Aschbrenner, K. A. \& Elwyn, G. Naturally occurring peer support through social media: the experiences of individuals with severe mental illness using YouTube. PLoS ONE 9, e110171 (2014).

51. Heinssen, R. K., Goldstein, A. B. \& Azrin, S. T. Evidence-based treatments for first episode psychosis: components of coordinated specialty care. White paper. Bethesda: National Institute of Mental Health. (2014).

52. Dixon, L. What it will take to make Coordinated Specialty Care available to anyone experiencing early schizophrenia: Getting over the hump. JAMA Psychiatry 74, 7-8 (2017).

(i) Open Access This article is licensed under a Creative Commons cc) Attribution 4.0 International License, which permits use, sharing, adaptation, distribution and reproduction in any medium or format, as long as you give appropriate credit to the original author(s) and the source, provide a link to the Creative Commons license, and indicate if changes were made. The images or other third party material in this article are included in the article's Creative Commons license, unless indicated otherwise in a credit line to the material. If material is not included in the article's Creative Commons license and your intended use is not permitted by statutory regulation or exceeds the permitted use, you will need to obtain permission directly from the copyright holder. To view a copy of this license, visit http://creativecommons. org/licenses/by/4.0/.

(c) The Author(s) 2019 